\section{EPV265/\#550 STRATEGIES TO MINIMISE MAJOR BLOOD LOSS IN PELVIC SURGERY - PELVIC VASCULATURE INTERVENTIONS}

${ }^{1} \mathrm{C}$ Downing, ${ }^{2} \mathrm{~S}$ Smyth*, 'M Alazzam. 'Oxford University Hospital NHS Foundation Trust, Gynaecology Oncology Department, Oxford, UK; ${ }^{2}$ Oxford University Hospitals NHS Foundation Trust, Gynaecological Oncology, Oxford, UK

\subsection{6/ijgc-2021-IGCS.336}

Objectives Intraoperative blood loss is a major contributing factor to morbidity and mortality in pelvic surgery. Therefore, the maintenance of haemostatic control is a crucial safety component.

Methods We performed a systematic review of the literature to identify current strategies to reduce blood loss in complex pelvic surgery.

Results This article reviews the current intra-operative strategies used to reduce bleeding. We discuss the surgical techniques for identifying the major pelvic arteries relevant to surgery. Once identified, various materials and equipment can be used to temporarily or permanently occlude these vessels with the aim of maintaining homeostasis. We review in which situations each option is best suited and the most appropriate apparatus for each approach. Correct surgical planning must also be determined to provide good visualisation of the pelvic field. Importantly, consideration must be given to the

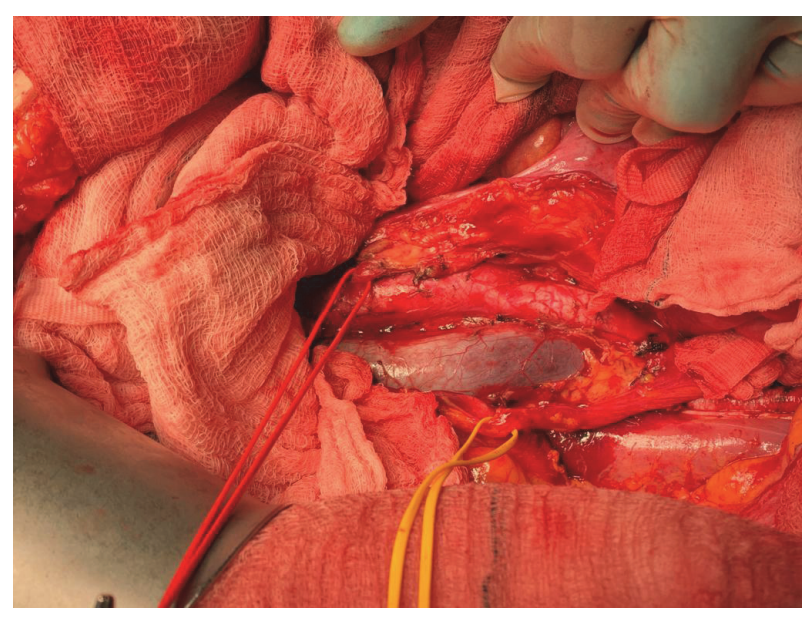

Abstract EPV265/\#550 Figure 1

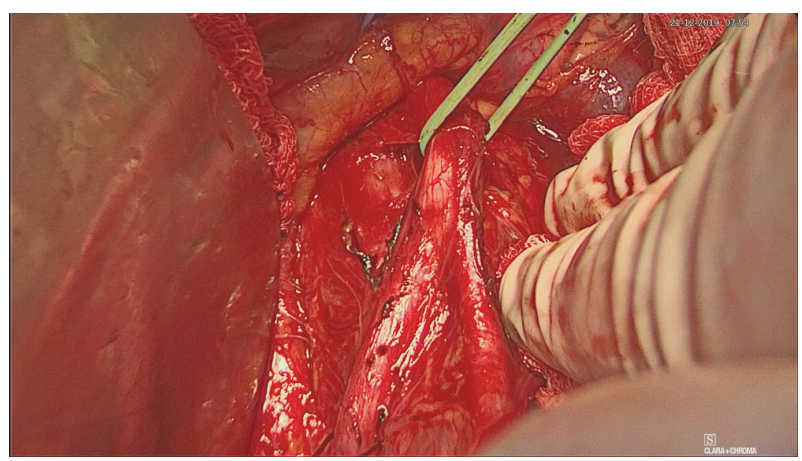

Abstract EPV265/\#550 Figure 2 vascularity and size of the lesion, which can vary greatly depending on the pathology. This will further impact on planning and dictate visualisation and securing of key vascular structures prior to manipulation of the lesion.

Conclusions In summary, adequate identification, access and manipulation of the appropriate vasculature is essential for prevention and management of major blood loss in pelvic surgery. Temporary and permanent occlusion of pelvic arteries has been used in practice for some time to manage haemostasis, yet further information is required to provide a definitive time interval as currently this area of research remains overlooked.

\section{EPV266/\#571 ACS NSQIP - PERSONALISED RISK PREDICTION TOOL FOR POSTOPERATIVE COMPLICATIONS IN GYNAEONCOLOGY SURGERY?}

${ }^{1} \mathrm{~L}$ Sevinyan*, ${ }^{2} \mathrm{~S}$ Jones, ${ }^{3} \mathrm{~J}$ Horne, ${ }^{3} \mathrm{R}$ Bharathan, ${ }^{1} \mathrm{~A}$ Tailor, ${ }^{1} \mathrm{~S}$ Butler-Manuel, ${ }^{4} \mathrm{P}$ Williams,

${ }^{1} \mathrm{~T}$ Madhuri. 'Royal Surrey Hospital NHS Foundation Trust, Gynaeoncology, Guildford, UK: ${ }^{2}$ Cardiff University, Gynaeoncology, Cardiff, UK; ${ }^{3}$ University Hospitals of Leicester NHS Trust, Gynaeoncology, Leicester, UK; ${ }^{4}$ University of Surrey, Medical Statistics, Guildford, UK

\subsection{6/ijgc-2021-IGCS.337}

Objectives The objective of our study was to explore the validity of ACS NSQIP (American College of Surgeons National Surgical Quality Improvement Program), a validated web-based tool based on 21 preoperative risk factors to predict 8 postoperative outcomes, in gynaeoncology for perioperative prediction of postoperative complications.

Despite the informed consent process, patients' understanding of complications is often limited, making it difficult to call the decision informed, so estimating the risk of postoperative complications is important for shared decision making.

Methods A retrospective multicentre cohort study evaluated 1552 patients who underwent surgery. Data collection undertaken through dedicated database and notes. Data collated on 764 patients undergoing robotic, 248 laparoscopic and 540 open surgery for gynaecological malignancy. Following data lock with the actual post-op event/complication, ACS NSQIP used to count predictive scores. Data analysis evaluating ACS-NSQIP validity and relevance in gynaeoncology patients and its ability to predict postoperative complications performed.

Results ACS-NSQIP was found to best predict mortality (AUC0.900), it was most accurate for prediction of complications as follows: discharge to rehabilitation (AUC-0.866), cardiac complications (AUC-0.844), sepsis (AUC-0.795), pneumonia (AUC-0.779), VTE (AUC-0.715), return to theatre (AUC0.715), surgical site infection (AUC-0.684), readmission (AUC0.680 ), renal failure (AUC-0.665). Poor result in the prediction of UTI (AUC-0.561) was noted.

Conclusions ACS-NSQIP risk calculator appears to predict major complications and post-operative mortality making it useful as an informed consent tool. Preliminary data suggests that further validation is required to fully evaluate if the risk scores may be used to inform patients pre-operatively of their risk of complications and is currently undertaken. 